\title{
Bayes Shrinkage Minimax Estimation in Inverse Gaussian Distribution
}

\author{
Gyan Prakash \\ Department of Community Medicine, Sarojini Naidu Medical College, Agra, India \\ E-mail: ggyanji@yahoo.com \\ Received November 19, 2010; revised May 14, 2011; accepted May 17, 2011
}

\begin{abstract}
In present paper, the properties of the Bayes Shrinkage estimator is studied for the measure of dispersion of an inverse Gaussian model under the Minimax estimation criteria.
\end{abstract}

Keywords: Bayes estimator, Bayes Shrinkage estimator, Uniformly Minimum Variance Unbiased Estimator (UMVUE), LINEX loss function (LLF) and Minimax Estimator

\section{Introduction}

The Inverse Gaussian distribution plays an important role in Reliability theory and Life testing problems. It has useful applications in a wide variety of fields such as Biology, Economics, and Medicine. It is used as an important mathematical model for the analysis of positively skewed data. The review article by Folks \& Chhikara $[1,2]$ and Seshadri [3] have proposed many interesting properties and applications of this distribution.

Let $x_{1}, x_{2}, \cdots, x_{n}$, be a random sample of size $n$, drawn from the inverse Gaussian distribution $\operatorname{IG}(\mu, \theta)$ : having probability density function

$$
\begin{aligned}
& f(x ; \mu, \theta)=\sqrt{\frac{\theta}{2 \pi x^{3}}} \exp \left(-\frac{\theta(x-\mu)^{2}}{2 \mu^{2} x}\right) ; \\
& x>0, \mu, \theta>0 .
\end{aligned}
$$

Here, $\mu$ stands for the mean and $\theta$ for the inverse measure of dispersion. The maximum likelihood estimates of $\mu$ and $\theta$ are given as:

$$
\hat{\mu}=\frac{1}{n} \sum_{i=1}^{n} x_{i}=\bar{x} \text { and } \hat{\theta}=\frac{n}{v},
$$

where

$$
v=\sum_{i=1}^{n}\left(\frac{1}{x_{i}}-\frac{1}{\bar{x}}\right) .
$$

The unbiased estimates of $\mu$ and $\theta$ are respecttively $\bar{x}$ and $\theta_{u}=\frac{1}{v}(n-3)$. Also, $\bar{x} \sim \operatorname{IG}(\mu, n \theta)$, $\theta v \sim \chi_{(n-1)}^{2}$ with $\bar{x}$ and $v$ being stochastically inde- pendent $([1,4,5]$,$) .$

Schuster [6] showed that

$\frac{\theta}{\mu^{2}} \sum_{i=1}^{n} \frac{\left(x_{i}-\mu\right)^{2}}{x_{i}}$ is distributed as chi-square distribution with $n$ degrees of freedom. If we assume that $\mu=\mu_{0}$ is known, the uniformly minimum variance unbiased (UMVU) estimator for measure of dispersion, $\theta^{-1}$ is

$$
U=\frac{1}{n} \sum_{i=1}^{n} \frac{\left(x_{i}-\mu_{0}\right)^{2}}{x_{i} \mu_{0}^{2}}
$$

and $n \theta \mathrm{U}$ follows a chi-square distribution with $n$ degrees of freedom.

The choice of the loss function may be crucial. It has always been recognized that the most commonly used loss function, squared error loss function (SELF) is in appropriate in many situations. If the SELF is taken as a measure of inaccuracy then the resulting risk is often too sensitive to the assumptions about the behavior of the tail of the probability distribution. In addition, in some estimation problems overestimation is more serious than the underestimation, or vice-versa [7]. To deal with such cases, a useful and flexible class of asymmetric loss function (LINEX loss function (LLF)) was introduced by Varian [8]. The reparameterized version of LLF ([9]) for any parameter $\theta$ is given as

$$
L(\Delta)=e^{a \Delta}-a \Delta-1 ; a \neq 0 \text { and } \Delta=(\hat{\theta}-\theta) / \theta .
$$

The sign and magnitude of ' $a$ ' represents the direction and degree of asymmetry respectively. The positive (negative) value of ' $a$ ' is used when overestimation is 
more (less) serious than underestimation. $L(\Delta)$ is approximately square error and almost symmetric if $|a|$ near to zero.

Thompson [10] suggested a procedure, which makes use of a prior information of the parameter in form of a guessed value by shrinking the usual unbiased estimator towards the guess value of the parameter with the help of a shrinkage factor $k(0 \leq k \leq 1)$. The experimenter according to his belief in the guess value specifies the values of shrinkage factor. The shrinkage estimator for the measure of dispersion $\theta^{-1}$ of $I G(\mu, \theta)$ when a guess value of $\theta$, say $\theta_{0}$, is available, is given by

$$
S=k \hat{\theta}+(1-k) \theta_{0}^{-1} ; 0 \leq k \leq 1 .
$$

Some shrinkage estimators for measure of dispersion $\theta^{-1}$ have been obtained by Pandey \& Malik [11] and have studied their properties under SELF-criterion. Prakash and Singh [12] have studied the properties of different shrinkage testimators for $\theta^{-1}$ under the LINEX loss function. Palmer [13] and Banerjee \& Bhattacharya [14] have discussed the Bayesian inference about the parameters of the inverse Gaussian distribution.

The present article proposed Bayes Shrinkage estimator based on the Minimax criteria for the measure of dispersion. A Bayes estimator for the measure of dispersion $\theta^{-1}$ under the vague prior has been obtained in the Section 2. Under the Minimax criteria the Bayes Minimax estimator has been obtained in the Section 3. A Shrinkage estimator construct by utilizing the Bayes Minimax estimator in the Section 4. Further, a numerical study has been presented in Section 5 and draws a conclusion about the Bayes Shrinkage Minimax estimator in Section 6.

\section{Bayes Estimator for Measure of Dispersion}

We are not going into debate or to justify the questions of the proper choice of the prior distribution. We consider a vague prior for the parameter $\theta$ which is an increasing function of the parameter $\theta$ and is given as

$$
g(\theta)=\theta^{d} ; d \geq 0 .
$$

Therefore, the posterior density of parameter $\theta$ is defined as

$$
\pi(\theta)=\frac{L(U ; \theta) \cdot g(\theta)}{\int L(U ; \theta) \cdot g(\theta) \mathrm{d} \theta} ; L(U ; \theta) \propto \theta^{\frac{n}{2}} e^{-\frac{n \theta U}{2}}
$$

After simplifying, the posterior density of parameter $\theta$ is obtained as

$$
\pi(\theta)=\left(\Gamma\left(\frac{n}{2}+d+1\right)\right)^{-1}\left(\frac{n U}{2}\right)^{\frac{n}{2}+d+1} \theta^{\frac{n}{2}+d} e^{-\frac{n \theta}{2} U} .
$$

The Bayes estimator for the measure of dispersion $\theta^{-1}$ under the LLF is obtained by simplifying the equality

$$
E_{p}\left(\theta e^{a \theta \hat{\theta}}\right)=e^{a} E_{p}(\theta) .
$$

Here, the suffix $p$ indicates that the expectation is taken under posterior density. After simplification the Bayes estimator for $\theta^{-1}$ under the LLF is

$$
\hat{\theta}=\phi U ; \phi=\frac{n}{2 a}\left(1-\exp \left(\frac{-2 a}{n+2 d+4}\right)\right) .
$$

\section{The Minimax Bayes Estimator}

The basic principle of this approach is to minimize the loss. The derivation depends primarily on a theorem, given by Hodge \& Lehmann [15] and can be stated as follows.

Let $\omega=\left\{F_{\theta}: \theta \in \Theta\right\}$ be a family of distribution functions and $C$ be a class of estimators of the parameter $\theta$. Suppose that $c^{*} \in C$ is a Bayes estimator against a prior distribution $g(\theta)$ on the parameter space $\Theta$. Then the Bayes estimator $c^{*}$ is said to be the Minimax estimator if the risk function of the estimator $c^{*}$ is independent on $\Theta$.

Here, the risk of the Bayes estimator $\theta$ given in (6) for the parameter $\theta^{-1}$ with respect to LLF is defined as

$$
R(\hat{\theta})=\int_{U} f(U) \cdot\left(e^{a \Delta}-a \Delta-1\right) \mathrm{d} U ; \Delta=\left(\hat{\theta}-\theta^{-1}\right) / \theta^{-1}(7)
$$

Since, $n \theta U$ is distributed as a Chi-square with $n$ degrees of freedom. Then by making a transformation $z=\frac{n \theta U}{2}$, the distribution of $z$ is obtained as

$$
f(z)=\left(\Gamma \frac{n}{2}\right)^{-1} e^{-z} z^{\frac{n}{2}-1} ; z>0
$$

Using equation (8) in the expression $R(\hat{\theta})$ we have

$R(\hat{\theta})=\frac{1}{\Gamma\left(\frac{n}{2}\right)} \int_{0}^{\infty} e^{-z} z^{\frac{n}{2}-1}\left\{e^{-a} e^{\frac{2 a \phi}{n} z}-\frac{2 a \phi}{n} z+a-1\right\} \mathrm{d} z$

$R(\hat{\theta})=e^{-a}\left(1-\frac{2 a \phi}{n}\right)^{-n / 2}-a(\phi-1)-1$.

The Equation (9) represents the risk of the Bayes estimator of the measure of dispersion, which is independent with the parameter $\theta$. Hence, the Bayes estimator $\theta$ is the Minimax estimator under the LLF loss criterion.

The following statistical problem (Minimax Estimation) is equivalent to some two person zero sum game between the Statistician (Player-II) and Nature (PlayerI). Here the pure strategies of Nature are the different 
values of $\theta$ in the interval $(0, \infty)$ and the mixed strategies of Nature are the prior densities of $\theta$ in the interval $(0, \infty)$. The pure strategies of Statistician are all possible decision functions in the interval $(0, \infty)$.

The expected value of the loss function is the risk function and it is the gain of the Player-I. Further, the Bayes risk is defined as

$$
R(\eta, \hat{\theta})=E_{\theta} R(\hat{\theta})
$$

Here, the expectation has been taken under the prior density of parameter $\theta$. If the loss function is continuous in both the estimator $\hat{\theta}$ and the parameter $\theta$, and convex in $\hat{\theta}$ for each value of $\theta$ then there exist measures $\eta^{*}$ and $\hat{\theta}^{*}$ for all $\theta$ and $\hat{\theta}$ so that, the following relation holds:

$$
R\left(\eta, \hat{\theta}^{*}\right) \leq R\left(\eta^{*}, \hat{\theta}^{*}\right) \leq R\left(\eta^{*}, \hat{\theta}\right)
$$

The number $R\left(\eta^{*}, \hat{\theta}^{*}\right)$ is known as the value of the game, and $\eta^{*}$ and $\hat{\theta}^{*}$ are the corresponding optimum strategies of the Player I and II. In statistical terms $\eta^{*}$ is the least favorable prior density of $\theta$ and the estimator $\hat{\theta}^{*}$ is the Minimax estimator. In fact, the value of the game is the loss of the Player-II. Hence, the optimum strategy of Player-II and the value of game are given for present case as

\begin{tabular}{lll}
\hline $\begin{array}{l}\text { Optimum } \\
\text { Strategy }\end{array}$ & $\begin{array}{l}\text { Corresponding } \\
\text { Loss }\end{array}$ & Value of Game \\
\hline$\hat{\theta}=\phi U$ & LLF & $e^{-a}\left(1-\frac{2 a \phi}{n}\right)^{-n / 2}-a(\phi-1)-1$ \\
\hline
\end{tabular}

\section{The Shrinkage Bayes Minimax Estimator}

Now, we construct a Shrinkage Bayes Minimax estimator as

$$
\bar{\theta}=\phi U+(1-\phi) \theta_{0}^{-1} .
$$

The risk of the Shrinkage Bayes Minimax estimator $\bar{\theta}$ under the LLF is obtain by using Equation (8) as

$$
\begin{aligned}
R(\bar{\theta}) & =\int_{\mathrm{U}} f(U) \cdot\left(e^{a \Delta^{\prime}}-a \Delta^{\prime}-1\right) \mathrm{d} U \\
R(\bar{\theta}) & =\exp (a \delta(1-\phi)-a) \cdot \exp \left(\frac{a n}{n+2 d+4}\right) \\
& -1+a(1-\delta)(1-\phi)
\end{aligned}
$$

where $\Delta^{\prime}=\left(\bar{\theta}-\theta^{-1}\right) / \theta^{-1}$ and $\delta=\theta \cdot \theta_{0}^{-1}$.

The comparison of the considered Shrinkage Bayes Minimax estimator $\bar{\theta}$ is performs with the help of a minimum class of estimator based on the UMVU estimator. Here, the considered class of estimator based on UMVU estimator is

$$
T=l U ; l \notin R^{+} .
$$

The risk of the estimator $T$ under the LINEX loss is given by

$$
R(T)=e^{-a}\left(1-\frac{2 a l}{n}\right)^{-n / 2}-a(l-1)-1 .
$$

The constant $l$, which minimizes $R(T)$ is given by

$$
l^{*}=\frac{n}{2 a}\left\{1-\exp \left(-\frac{2 a}{n+2}\right)\right\} .
$$

Thus, the improved estimators among the class $T$ is

$$
T^{*}=l^{*} U
$$

with the risk under the LLF is given as

$$
R\left(T^{*}\right)=\left(\frac{n}{2}+1\right)\left(\exp \left(-\frac{2 a}{n+2}\right)-1\right)+a .
$$

Remark: It observed that the value of $\varphi$ is lies between zero and one for the selected parametric set of values which are considered later for the numerical findings. Therefore, $\varphi$ is considered as the shrinkage factor.

\section{A Numerical Study}

The relative efficiencies for the Shrinkage Bayes Minimax estimator $\bar{\theta}$ relative to the improved estimator $T^{*}$ is defined as

$$
R E\left(\bar{\theta}, T^{*}\right)=\frac{R\left(T^{*}\right)}{R(\bar{\theta})} .
$$

The relative efficiencies are the functions of $n, a$, $\delta$ and $d$. For the selected set of values of $n=05,10,15,25 ; \quad a= \pm 0.25, \pm 0.50 ; \quad \delta=0.25(0.25) 1.75$ and $d=0.25,0.50,01,1.50,02,05$; the relative efficiency have been calculated. The numerical findings are presented here only for $n=05$ and $n=15$ in the Tables 1 and $\mathbf{2}$ respectively.

It is observed that the Shrinkage Bayes Minimax estimator $\bar{\theta}$ is performs better then the improved estimator $T^{*}$ for the all selected parametric set of values for $0.25<\delta<1.75$. Further, as sample size $n$ increases the relative efficiency decreases for all considered parametric set of values and attains maximum efficiency at the point $\delta=1$. Further, it is also observed that the relative efficiency increases as $d$ increases when $\delta$ lie between $0.50<\delta<1.50$. It is seen also that, as 'a' increase relative efficiency first increases for $\delta \leq 0.75$ and then decrease for the other values of $\delta$.

\section{Conclusions}

In present paper we obtained the Shrinkage estimator 
based on the Minimax estimation criteria for the measure of dispersion of the inverse Gaussian distribution. We ob- served that on the basis of the relative efficiency, the proposed Shrinkage Bayes Minimax estimator $\bar{\theta}$ performs

Table 1. Relative efficiency for the estimator $\bar{\theta}$ with respect to $T^{*}$ under LLF for $n=5$ and different values of $a$, $d$ and $\delta$.

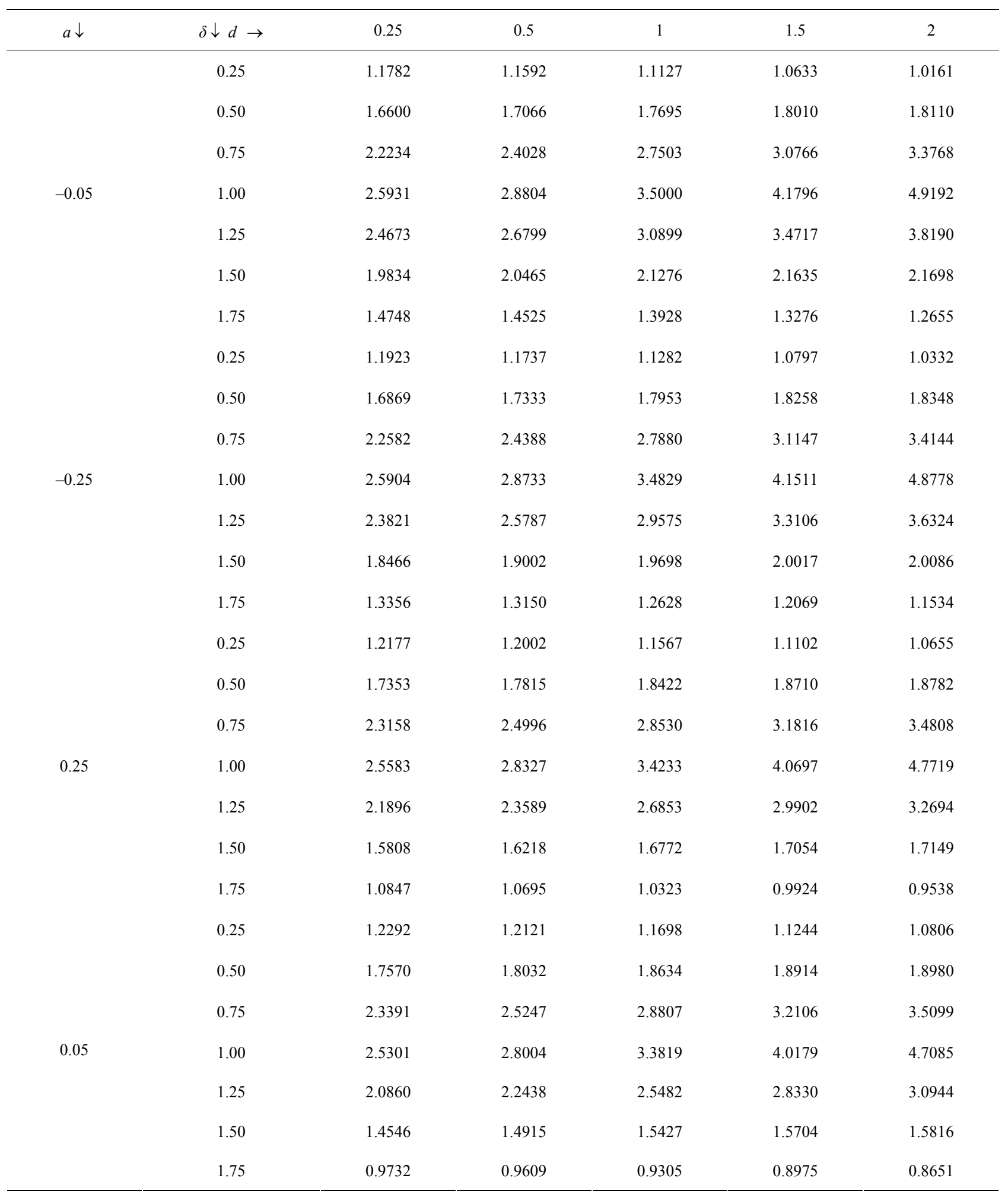


Table 2. Relative efficiency for the estimator $\bar{\theta}$ with respect to $T^{*}$ under LLF for $n=15$ and different values of $a, d$ and $\delta$.

\begin{tabular}{|c|c|c|c|c|c|c|}
\hline$a \downarrow$ & $\delta \downarrow d \rightarrow$ & 0.25 & 0.5 & 1 & 1.5 & 2 \\
\hline \multirow{7}{*}{-0.05} & 0.25 & 1.0624 & 1.0484 & 1.0109 & 0.9659 & 0.9182 \\
\hline & 0.50 & 1.2457 & 1.2660 & 1.2949 & 1.3099 & 1.3134 \\
\hline & 0.75 & 1.4014 & 1.4590 & 1.5717 & 1.6804 & 1.7841 \\
\hline & 1.00 & 1.4912 & 1.5691 & 1.7308 & 1.9004 & 2.0780 \\
\hline & 1.25 & 1.4755 & 1.5432 & 1.6762 & 1.8048 & 1.9277 \\
\hline & 1.50 & 1.3723 & 1.4048 & 1.4538 & 1.4835 & 1.4967 \\
\hline & 1.75 & 1.2137 & 1.2068 & 1.1766 & 1.1323 & 1.0812 \\
\hline \multirow{7}{*}{-0.25} & 0.25 & 1.0718 & 1.0584 & 1.0219 & 0.9777 & 0.9308 \\
\hline & 0.50 & 1.2612 & 1.2825 & 1.3130 & 1.3291 & 1.3335 \\
\hline & 0.75 & 1.4171 & 1.4759 & 1.5911 & 1.7021 & 1.8080 \\
\hline & 1.00 & 1.4875 & 1.5658 & 1.7285 & 1.8991 & 2.0777 \\
\hline & 1.25 & 1.4557 & 1.5196 & 1.6447 & 1.7654 & 1.8807 \\
\hline & 1.50 & 1.3262 & 1.3532 & 1.3930 & 1.4159 & 1.4246 \\
\hline & 1.75 & 1.1480 & 1.1375 & 1.1038 & 1.0595 & 1.0107 \\
\hline \multirow{7}{*}{0.25} & 0.25 & 1.0895 & 1.0773 & 1.0428 & 1.0004 & 0.9548 \\
\hline & 0.50 & 1.2898 & 1.3132 & 1.3470 & 1.3656 & 1.3718 \\
\hline & 0.75 & 1.4427 & 1.5043 & 1.6247 & 1.7407 & 1.8513 \\
\hline & 1.00 & 1.4875 & 1.5645 & 1.7244 & 1.8920 & 2.0674 \\
\hline & 1.25 & 1.4012 & 1.4580 & 1.5689 & 1.6757 & 1.7775 \\
\hline & 1.50 & 1.2222 & 1.2406 & 1.2665 & 1.2796 & 1.2822 \\
\hline & 1.75 & 1.0133 & 0.9990 & 0.9631 & 0.9215 & 0.8782 \\
\hline \multirow{7}{*}{0.05} & 0.25 & 1.0979 & 1.0862 & 1.0527 & 1.0112 & 0.9664 \\
\hline & 0.50 & 1.3031 & 1.3274 & 1.3630 & 1.3829 & 1.3900 \\
\hline & 0.75 & 1.4528 & 1.5158 & 1.6390 & 1.7577 & 1.8708 \\
\hline & 1.00 & 1.4803 & 1.5569 & 1.7158 & 1.8825 & 2.0568 \\
\hline & 1.25 & 1.3675 & 1.4210 & 1.5257 & 1.6263 & 1.7221 \\
\hline & 1.50 & 1.1660 & 1.1812 & 1.2021 & 1.2119 & 1.2127 \\
\hline & 1.75 & 0.9462 & 0.9313 & 0.8959 & 0.8566 & 0.8164 \\
\hline
\end{tabular}

better than an improved estimator in a wide range of $\delta$ which is defined here as the ratio between the true value and guess (prior point) value of the unknown parameter under the LLF. Thus, we suggest using the Minimax estimator under LLF for estimating the measure of dispersion under the Shrinkage setup.

\section{References}

[1] J. L. Folks and R. S. Chhikara, "The Inverse Gaussian
Distribution and Its Statistical Application-A Review," Journal of the Royal Statistical Society, Vol. 40, No. 3, 1978, pp. 263-289.

[2] J. L. Folks and R. S. Chhikara, "The Inverse Gaussian Distribution," Marcel Dekker, New York, 1989.

[3] V. Seshadri, "The Inverse Gaussian Distribution," Statistical Theory and Applications, Springer-Verlag, New York, 1998.

[4] M. C. K. Tweedie, "Statistical Properties of Inverse Gaussian Distribution-I," The Annals of Mathematical Statis- 
tics, Vol. 28, No. 2, 1957, pp. 362-377. doi:10.1214/aoms/1177706964

[5] M. C. K. Tweedie, "Statistical Properties of Inverse Gaussian Distribution-II," The Annals of Mathematical Statistics, Vol. 28, No. 2, 1957, pp. 696-705. doi:10.1214/aoms/1177706881

[6] J. J. Schuster, "On the Inverse Gaussian Distribution," Journal of American Statistical Association, Vol. 63, No. 324, 1968, pp. 1514-1516. doi:10.2307/2285899

[7] A. Parsian and S. N. U. A. Kirmani, "Estimation under LINEX Loss Function,” In: A. Ullah, A. T. K. Wan, A. Chaturvedi and M. Dekker, Eds., Handbook of Applied Econometrics and Statistical Inference, CRC Press, Boca Raton, 2002, pp. 53-76.

[8] H. R. Varian, "A Bayesian Approach to Real Estate Assessment," In: S. E. Feinberge and A. Zellner, Eds., Studies in Bayesian Econometrics and Statistics, Amsterdam North Holland, 1975, pp. 195-208.

[9] D. C. Singh, G. Prakash and P. Singh, "Shrinkage Testimators for the Shape Parameter of Pareto Distribution Using the Linex Loss Function," Communication in Statistics: Theory and Methods, Vol. 36, No. 4, 2007, pp. 741-753. doi:10.1080/03610920601033694

[10] J. R. Thompson, "Some Shrinkage Techniques for Esti- mating the Mean," Journal of the American Statistical Association, Vol. 63, No. 321, 1968, pp. 113-122. doi: $10.2307 / 2283832$

[11] B. N. Pandey and H. J. Malik, "Some Improved Estimators for a Measure of Dispersion of an Inverse Gaussian Distribution," Communications in Statistics: Theory and Methods, Vol. 17, 1988, pp. 3935-3949. doi:10.1080/03610928808829847

[12] G. Prakash and D. C. Singh, "Shrinkage Testimators for the Inverse Dispersion of the Inverse Gaussian Distribution under the Linex Loss Function," Austrian Journal of Statistics, Vol. 35, No. 4, 2006, pp. 463-470.

[13] T. Palmer, "Certain Non-Classical Inference Procedures Applied to the Inverse Gaussian Distribution," Ph.D. Dissertation, Oklahoma State University, Stillwater, 1973.

[14] A. K. Banerjee and G. K.Bhattacharya, "Bayesian Results for the Inverse Gaussian Distribution with an Application," Technometrics, Vol. 21, No. 2, 1979, pp. 247-251. doi: $10.2307 / 1268523$

[15] J. I. Hodge and E. L. Lehmann, "Some Problems in Minimax Estimation," Annals of Mathematical Statistics, Vol. 21, No. 2, 1950, pp. 182-197. doi:10.1214/aoms/1177729838 